\title{
HIV and History Taking
}

\author{
Ashfaq UI Hassan* and Zahida Rasool
}

SKIMS Medical College, Srinagar, Kashmir, India

\begin{abstract}
The need for medical students, Primary health care physicians, general practitioners to take an effective sexual history in a decent manner poses a challenge .It is in certain circumstances very difficult to go into details of sexual history while at the same time maintaining an effective doctor patient relationship. To reduce the incidence and prevalence of HIV infections and other diseases associated with this dreadful disease our medical students, doctors should be well prepared for the art of asking questions directed at individuals' most personal details.

By controlling the occurrence of HIV infections, one is not controlling Epidemic of AIDS only but also the associated host of diseases seen arising as a result of AIDS. The cost of treating HIV positive individuals in the society is immense and simple control of this infection relieves the society of the huge economic burden. The role of history taking can never be underestimated.
\end{abstract}

Keywords: AIDS; Screening; History; Sexually transmitted diseases; Sexual practices

\section{Introduction}

Effective drugs, modern diagnostic techniques, Multidisciplinary approach are essential for combating this deadly disease but the old saying prevention is better than cure seems to be the best answer in controlling the spread and its consequences. There is still much that needs to be done to foster HIV prevention in primary care. Doctor's way of history taking raises questions as to whether society can count on primary care physicians to assume their part of responsibility in controlling the AIDS epidemic. Developing a system to identify persons most in need of HIV prevention services is a key step in implementing the sort of prevention approach such as the Serostatus Approach to Fighting the HIV Epidemic (SAFE).

A sexual history is important for all patients to provide information that identifies those at risk for sexually transmitted diseases, including HIV, to guide risk reduction counseling, and to identify what anatomic sites are suitable for STD screening. This basic sexual history tool can be used by clinicians as a guide to determine the patient's risk for STDs. This history can be taken by the clinician as part of the history and physical, or done by the patient as a self-administered questionnaire. In different parts of the world the approach should be different but the results of the history taking in all cases should be clear cut as to the likely outcome of whether an individual has been a potential candidate likely to be affected by the HIV virus and if he is a source of danger to others.

\section{Questionnaire}

The questionnaire template may not be culturally appropriate for some patients, and it can be adjusted as needed.

Despite effective worldwide campaign against HIV in all parts of the world including underdeveloped world, developing world and developed world. Each year there are more and more new HIV infections, which shows that people either aren't learning the message about the dangers of HIV, or are unable or unwilling to act on it. Many people are dangerously ignorant about the virus, with surveys around the world showing alarmingly low levels of awareness and understanding about HIV amongst many groups. Education can help to overcome such ignorance, and thereby prevent HIV infections from occurring.

\section{Text}

Education needs to be an ongoing process to prevent new infections from taking place. This can be seen as consisting of two processes: firstly, giving people information about HIV and AIDS, such as how they are transmitted and how people can protect themselves from infection. Secondly, people must be taught how to put this information to use and act on it practically - how to get and use condoms, how to suggest and practice safer sex, how to prevent infection in a medical environment or when injecting drugs.

\section{Who needs to be educated?}

- People who have not yet been educated and may be at risk of becoming infected.

- People who have already been educated for whom the education was not effective.

- People who are already infected.

- People who are at high risk.

The doctor remains an important source within the community for addressing HIV/AIDS prevention and early detection. Doctors should be acquainted with the knowledge and practices relating to HIV prevention education, risk assessment, and case finding

Doctors play a major role in structuring comprehensive patient management and community support systems which are a critical part of the multidisciplinary model of AIDS care

\section{Introducing yourself as a doctor}

Introducing yourself as a doctor forms the first impression with

*Corresponding author: Ashfaq UI Hassan, Physician Directorate of Health Services, SKIMS Medical College, Srinagar, Kashmir, India, E-Mail: ashhassan@rediffmail.com

Received November 28, 2012; Accepted January 05, 2013; Published January 10, 2013

Citation: UI Hassan A, Rasool Z (2013) HIV and History Taking. J AIDS Clin Res 4 190. doi:10.4172/2155-6113.1000190

Copyright: (c) 2013 UI Hassan A, et al. This is an open-access article distributed under the terms of the Creative Commons Attribution License, which permits unrestricted use, distribution, and reproduction in any medium, provided the original author and source are credited. 
your patient. Starting with a battery of questions without proper introduction would be an irrational approach to start.After formal introduction a doctor can start as:

"I am going to take a few minutes to ask you some direct question about your sexual health. These questions are very personal, but it is important for me to know so I can help you be healthy. I ask these questions to all of my patients regardless of age or marital status and they are just as important as other questions about your physical and mental health. Like the rest of this visit, this information is strictly confidential."

\section{Questions should emphasize upon}

The 4 "P"s:

1. Partners,

2. Sexual Practices,

3. Past STDs, and

4. Protection from STDs

\section{Additional questions to identify HIV and hepatitis risk}

Immunization history for hepatitis A and B can be noted at this point, as well as past HIV testing. Hepatitis A and B immunization is recommended.

- "Have you ever got the hepatitis B vaccine (all 3 doses)?"

- "Have you ever got hepatitis A vaccine (2 doses)?"

- "Have you ever been tested for HIV, the virus that causes AIDS?"

\section{Finishing up}

By the end of this section of the interview, the patient may have come up with information or questions that he was not ready to discuss earlier.

- "Is there anything else about your sexual practices that I need to know about to ensure you good health care?"

- "Do you have any questions?"

\section{Targeting High Risk Groups}

- A variety of demographic, behavioral, and social factors place people at risk for becoming infected with HIV.

- Anyone of any age who engages in unprotected oral, vaginal, or anal intercourse with anyone other than an uninfected, mutually monogamous partner

- Anyone whose partner engages in unprotected intercourse with others

- Drug users who share needles and other drug works

- Anyone who receives an injection with a potentially contaminated needle or syringe

- The sexual partner(s) of an injection drug user

- Recipients of transfusions or treatment with blood or blood products in regions where reliable screening of the blood supply does not occur

- Anyone who uses potentially contaminated tattoo needles or other skin-piercing instruments Any workers or clients at health care facilities who come into contact with blood, blood products, unclean needles, or surgical instruments A fetus or nursing child of a mother who is infected with HIV

Some factors that affect social vulnerability include gender inequalities, economic power, youth, cultural constructs, and government policies.

A doctor can be faced with patients reluctant to disclose their sexual behaviors in the beginning. But on subsequent interviews making the individual realizes the dangers he/she is faced with as a result of HIV infection, he/she can ultimately confess. The art of identifying potentially infected individuals who are reluctant to disclose initially and extracting vital clues in this regard is no more than an art of a good doctor. This implies that in certain cases more than one round of interview with an individual might be needed to extract all relevant information about the sexual history of an individual. 